\title{
Homeopathic Prevention and Management of Epidemic Diseases
}

\author{
Jennifer Jacobs ${ }^{1}$ \\ ${ }^{1}$ Department of Epidemiology, School of Public Health and \\ Community Medicine, University of Washington, Seattle, \\ Washington, United States
}

Homeopathy 2018;107:157-160.

\begin{abstract}
Address for correspondence Jennifer Jacobs, MD, MPH, Department of Epidemiology, School of Public Health and Community Medicine, University of Washington, Seattle, WA, United States (e-mail: jjacobs@igc.org).
\end{abstract}

\begin{abstract}
Keywords

- homeopathy

- epidemic diseases

- homeoprophylaxis

- nosodes

- isopathy

- genus epidemicus

Background Homeopathy has been used to treat epidemic diseases since the time of Hahnemann, who used Belladonna to treat scarlet fever. Since then, several approaches using homeopathy for epidemic diseases have been proposed, including individualization, combination remedies, genus epidemicus, and isopathy.

Methods The homeopathic research literature was searched to find examples of each of these approaches and to evaluate which were effective.

Results There is good experimental evidence for each of these approaches. While individualization is the gold standard, it is impractical to use on a widespread basis. Combination remedies can be effective but must be based on the symptoms of a given epidemic in a specific location. Treatment with genus epidemicus can also be successful if based on data from many practitioners. Finally, isopathy shows promise and might be more readily accepted by mainstream medicine due to its similarity to vaccination. Conclusion Several different homeopathic methods can be used to treat epidemic diseases. The challenge for the future is to refine these approaches and to build on the knowledge base with additional rigorous trials. If and when conventional medicine runs out of options for treating epidemic diseases, homeopathy could be seen as an attractive alternative, but only if there is viable experimental evidence of its success.
\end{abstract}

\section{Introduction}

Epidemics are the scourge of human civilization. A smallpox epidemic in Greece in 430 BC that killed more than 30,000 people was one of the first-documented epidemics. Throughout recorded history there have been reports of epidemics-from the plague in Europe in the Middle Ages to the smallpox that killed millions of native peoples in the Americas after the arrival of Europeans in the new world. Yellow fever struck America in the late 19th century and the worldwide flu pandemic of 1918 and 1919 is estimated to have killed 20 to 40 million people. More recently, there have been epidemics of HIV-AIDS, Ebola, swine flu, bird flu, and Zika virus among others. Cholera and other infectious diseases continue to be a threat wherever large numbers of people are displaced into refugee camps.
One of the most important challenges of public health is to control these epidemic outbreaks, which are diseases that spread rapidly and widely, affecting many individuals in a population at the same time. Vaccines, anti-viral medications, and antibiotics are the standard conventional treatments for these diseases. However, difficulties with developing and disseminating immunizations, viral mutations, and the growing problem of antibiotic-resistant bacteria are undermining these approaches. The World Health Organization has recently called for "innovative" approaches for treating communicable diseases.

The use of homeopathy for epidemic diseases dates back to the time of Hahnemann, who recommended Belladonna for scarlet fever. It is interesting to note that homeopathy was invented in the same year as Jenner first practiced received

October 24, 2017

accepted after revision

March 28, 2018

published online

May 12, 2018
Copyright $\odot 2018$ The Faculty of Homeopathy
DOI https://doi.org/ 10.1055/s-0038-1649487. ISSN $1475-4916$. 
vaccination (1796) and that Hahnemann strongly favored vaccination. Homeopathy first rose to prominence in the 19 th century due to its success in treating epidemic diseases such as cholera, yellow fever, and typhus. There are several different strategies that have been used by homeopaths to treat epidemic diseases, including individualization, combination remedies, genus epidemicus, and isopathy. We will look at the evidence for each of these in this article.

\section{Individualization}

The gold standard in homeopathic practice is individualization. This requires an interview by a trained homeopathic prescriber to determine the specific remedy for each person, based on the totality of physical, emotional, and mental symptoms. We have ample evidence of the effectiveness of individualized homeopathy for epidemic diseases. With my own team, we successfully treated children with acute diarrhea in both Nicaragua and Nepal, using individualized single homeopathic remedies. ${ }^{1,2}$ In Nicaragua, 18 different remedies were used, while 14 were prescribed individually in Nepal. Other homeopathic researchers have reported treating other epidemic diseases, such as Chikungunya fever, pulmonary tuberculosis, and malaria, using individualized prescribing. ${ }^{3-5}$

The main drawbacks of individualized treatment of epidemic diseases are related to logistics and cost. Except potentially in a few countries of the world with large numbers of homeopaths, such as India and Brazil, there are not enough trained prescribers to treat everyone individually during a full-scale epidemic. The cost of mobilizing such a force would be considerable. Another problem is the accuracy of the prescription, which can vary depending on the skill of the prescriber. Because of this, researchers interested in using homeopathy to treat epidemic illnesses have sought other strategies.

\section{Combination Remedies}

Combining several different remedies that are commonly prescribed for a specific disease into one medication could be a cost-effective way to treat people during an epidemic. There have been several studies to evaluate this approach, some more successful than others. Because of the success of the Nepal and Nicaragua childhood diarrhea studies, our team performed a clinical trial in Honduras using a combination remedy composed of the five most common single remedies, which were prescribed in $80 \%$ of the cases in Nicaragua and Nepal. ${ }^{6}$ These were Arsenicum album, Calcarea carbonica, Chamomilla, Podophyllum, and Sulfur. The results of this study were disappointing-we found no difference between those who received the homeopathic combination and those who were given a placebo. In retrospect, it is possible that the symptoms of the children in Honduras or the pathological agents involved were different than those of the previous studies and required a different combination of homeopathic remedies. A pilot study to determine the most common remedies for Honduran children with diarrhea might have led to a combination remedy with better results.

A team in Pakistan recently reported the results of a trial of a homeopathic combination remedy for dengue fever. ${ }^{7}$
Dengue is a mosquito-borne, flu-like illness caused by a virus and is endemic in more than 110 countries. It infects 50 to 100 million people each year and causes as many as 25,000 deaths. The study compared a combination of ten different remedies known to be indicated for symptoms of dengue fever with standard supportive therapy as recommended by the World Health Organization. The remedies used were Bryonia alba, Rhus toxicodendron, Gelsemium, Aconitum napellus, Eupatorium perfoliatum, China boliviana, Hamamelis, Citrullus colocynthis, Crotalus horridus, and Phosphorus. Supportive treatments used were acetaminophen (paracetamol), fluid replacement, and medications for nausea and vomiting. At the end of 6 days, there were statistically significant improvements in blood platelet levels, hematocrit, and white blood cell counts in the homeopathic combination group compared with supportive therapy. No information was given about the clinical course of the disease in the two treatment groups.

This study is in contrast to another by our group that compared a combination of six remedies with placebo in patients with symptoms of dengue fever in Honduras. ${ }^{8}$ Based on materia medica study, the remedies included in the combination were Aconitum napellus, Belladonna, Bryonia alba, Eupatorium perfoliatum, Gelsemium, and Rhus toxicodendron. We found no differences in the resolution of pain and fever or the use of analgesics between the two groups. Viral testing revealed that only 3 of the 60 patients in our study had laboratory-confirmed dengue. Even so, one would expect that patients with pain and fever would respond to this combination of remedies that are well known for treating flu-like syndromes. Again, it is possible that the composition of the combination remedy did not accurately match the symptoms of the patients treated.

As we can see from these studies, the difficulty with combination remedies is in matching the correct remedies with the epidemic being treated. The best approach in the future would be first to treat patients in a specific epidemic with individualized remedies and then to incorporate the most commonly prescribed of these into a combination remedy.

\section{Genus Epidemicus}

The genus epidemicus is the remedy found to be most effective for a particular epidemic once data have been gathered from several cases. This concept was first put forth by Samuel Hahnemann in the Organon of Medicine, Aphorism 241 , as “...each single epidemic is of a peculiar, uniform character common to all the individuals attacked, and when this character is found in the totality of the symptoms common to all, it guides us to the discovery of homoeopathic (specific) remedy suitable for all the cases...."9

There is anecdotal evidence that homeopathy was successful during the Spanish flu epidemic of 1918 to 1919, in which at least 20 million people died worldwide, more than 500,000 in the United States alone. According to the historian Julian Winston, the death rates for patients treated with homeopathy were 1 to $2 \%$ compared with a 30 to $60 \%$ mortality for those treated by conventional physicians. ${ }^{10}$ 
The genus epidemicus for the Spanish flu, according to Winston, was Gelsemium.

A modern example of the use of genus epidemicus was reported by a group in India that performed a double-blind placebo-controlled trial using Bryonia alba to prevent Chikungunya fever during an epidemic of this viral disease in 2007. ${ }^{11}$ Following the method of Hahnemann, investigators first repertorized the symptoms of 205 patients with fever and arthralgia, characteristic of Chikungunya. A group of homeopathic experts then came to a consensus for Bryonia as the genus epidemicus after confirming symptoms in the materia medica.

Family groups of approximately 200 individuals, known as "clusters," were then randomized to take either Bryonia 30C or placebo during the time of the active epidemic. Out of nearly 20,000 healthy individuals in each group, $12.8 \%$ of those receiving Bryonia developed the illness compared with $15.8 \%$ of those taking the placebo $(p=0.03)$. This translates into a relative risk reduction of nearly $20 \%$ in contracting Chikungunya. These results are promising and hopefully will encourage more researchers to evaluate this approach.

\section{Isopathy}

Isopathic remedies are made from the actual cause of the illness, or from its byproducts, to treat that same condition. It is somewhat akin to conventional vaccination, although the preparation is made in the homeopathic manner of potentization, with repeated dilutions and shaking (succussion) at each step. The particular agent can be a nosode, made from diseased tissues or bacteria, such as Tuberculinum from tuberculosisinfected lung tissue, or Anthracinum from anthrax poison. Or, it can be a sarcode, made from a healthy tissue or organ, such as Oscillococcinum, derived from duck heart and liver. Isopathic remedies can also be made from toxic chemicals, such as arsenic and mustard gas, allergy-causing agents, such as mixed-grass pollens, and potentized drugs, such as penicillin, to treat the adverse effects of that medication.

One of the first experiments in isopathy took place in London and Glasgow during World War II, using potentized mustard gas as a preventative for chemical injury from mustard gas. ${ }^{12}$ Results of this experiment demonstrated a statistically significant decrease in deep skin lesions in those given mustard gas 30C prior to applying mustard gas to their skin compared with those receiving a placebo. Another study, using an isopathic preparation of mixed-grass pollens to treat patients with allergic rhinitis, also found significant results. ${ }^{13}$

There are several interesting studies using isopathy to treat epidemic diseases. As previously noted, Oscillococcinum is a homeopathic preparation made from duck heart and liver, thought by some to be a reservoir of influenza virus, although this has not been definitively proven. Oscillococcinum has been used successfully in a double-blind study to treat patients with influenza-like illness. ${ }^{14}$ In another experiment, a nosode was made from tissues of mice infected with the bacterium causing tularemia, a tick-borne disease mostly found in animals but sometimes in humans. ${ }^{15}$ Potencies of $30 \mathrm{C}, 200 \mathrm{C}$, or $1 \mathrm{M}$ were given prophylactically to healthy mice for 30 days. Age-matched animals were treated during the same time with $70 \%$ ethanol as controls. After a challenge with a lethal dose of the bacterium, all those treated with the nosode, except those receiving the $1 \mathrm{M}$, had statistically significant decreased mortality and increased mean times to death. The overall mortality rate was reduced by $22 \%$ in those treated with these nosodes.

A striking example of the use of isopathy for disease prevention occurred in Cuba during an epidemic of leptospirosis from 2007 to $2008 .^{16}$ Caused by a gram-negative bacterium, leptospirosis is spread through the urine of domestic and wild animals and can cause serious infections such as meningitis, hepatitis, and pneumonitis. It is especially prevalent in areas with poor sanitary conditions and can reach epidemic proportions after heavy rainfall and flooding from hurricanes and other natural weather disasters.

During a period of widespread flooding and increased incidence of leptospirosis in three provinces of Cuba in 2007, $97 \%$ of the entire population over 1 year of age was treated with a nosode that comprised four strains of inactivated Leptospira. ${ }^{17}$ Over a 5 -week period, more than 2 million people received two oral doses of the $200 \mathrm{C}$ potency of this preparation. This was followed nearly a year later with two $10 \mathrm{M}$ doses of the same nosode. Disease surveillance statistics revealed an $84 \%$ decreased incidence of the disease in these provinces in 2008 compared with previous years, despite the occurrence of three large hurricanes. In contrast, the incidence of leptospirosis in the other, untreated, provinces of Cuba rose by $21.7 \%$. There is a concern in the leptospirosis data, however, that the incidence of the disease dropped from the highest to the lowest in successive weeks: between week 52 of 2007 and week 1 of 2008 .

\section{Summary and Conclusions}

As we have seen from these examples, homeopathy has been used for more than a century in many parts of the world to treat epidemic diseases. While individualization is the gold standard, it is impractical to use on a widespread basis. Combination remedies can be effective but must be based on the symptoms of a given epidemic in a specific location. Treatment with genus epidemicus can also be successful if based on data from many practitioners. Finally, isopathy shows promise and might be more readily accepted by mainstream medicine due to its similarity to vaccination.

Whether using individualization, combination remedies, genus epidemicus, or isopathy, we have several good studies documenting positive results with homeopathy. The challenge for the future is to refine these approaches and to build on the knowledge base with additional rigorous trials. If and when conventional medicine runs out of options for treating epidemic diseases, homeopathy could be seen as an attractive alternative, but only if there is viable experimental evidence of its success.

Highlights

- Homeopathy has been used for over 200 years to treat epidemic diseases, beginning with Samuel Hahnemann prescribing Belladonna for scarlet fever.

- There are four main approaches of using homeopathy for epidemic disease: individualization, combination remedies, genus epidemicus, and isopathy. 
- There is experimental evidence of effectiveness of each of these approaches.

- More research needs to be performed to enlarge the research base and to make homeopathy an attractive treatment alternative for epidemic diseases.

\section{Conflict of Interest}

None.

\section{References}

1 Jacobs J, Jiménez LM, Gloyd SS, Gale JL, Crothers D. Treatment of acute childhood diarrhea with homeopathic medicine: a randomized clinical trial in Nicaragua. Pediatrics 1994;93:719-725

2 Jacobs J, Jiménez LM, Malthouse S, et al. Homeopathic treatment of acute childhood diarrhea: results from a clinical trial in Nepal. J Altern Complement Med 2000;6:131-139

3 Wadhwani GG. Homeopathic drug therapy. Homeopathy in Chikungunya fever and post-Chikungunya chronic arthritis: an observational study. Homeopathy 2013;102:193-198

4 Chand KS, Manchanda RK, Mittal R, Batra S, Banavaliker JN, De I. Homeopathic treatment in addition to standard care in multi drug resistant pulmonary tuberculosis: a randomized, double blind, placebo controlled clinical trial. Homeopathy 2014;103:97-107

5 van Erp VMA, Brands M. Homoeopathic treatment of malaria in Ghana: open study and clinical trial. Br Homeopath J 1996;85:66-70

6 Jacobs J, Guthrie BL, Montes GA, et al. Homeopathic combination remedy in the treatment of acute childhood diarrhea in Honduras. J Altern Complement Med 2006;12:723-732

7 Saeed-ul-Hassan S, Tariq I, Khalid A, Karim S. Comparative clinical study on the effectiveness of homeopathic combination remedy with standard maintenance therapy for dengue fever. Trop J Pharm Res 2013;12:767-770

8 Jacobs J, Fernandez EA, Merizalde B, Avila-Montes GA, Crothers D. The use of homeopathic combination remedy for dengue fever symptoms: a pilot RCT in Honduras. Homeopathy 2007;96:22-26

9 Hahnemann S. Organon of Medicine. 6th ed. New Delhi: B Jain Publishers; 2004:267

10 Winston J. The Faces of Homeopathy. Wellington, New Zealand: Great Auk Publishing; 1999

11 Nair KRJ, Gopinadhan S, Kurup TNS, et al. Homoeopathic Genus Epidemicus 'Bryonia alba' as a prophylactic during an outbreak of Chikungunya in India: a cluster-randomised, double-blind, placebo- controlled trial. Indian J Res Homoeopathy 2014; 8:160-165

12 Paterson J, Wheeler C, Cooper LH, et al. Report on mustard gas experiments (Glasgow and London). Br Homeopath J 1943;33:1-12

13 Reilly DT, Taylor MA, McSharry C, Aitchison T. Is homoeopathy a placebo response? Controlled trial of homoeopathic potency, with pollen in hayfever as model. Lancet 1986;ii:881-886

14 Ferley JP, Zmirou D, D'Adhemar D, Balducci F. A controlled evaluation of a homoeopathic preparation in the treatment of influenza-like syndromes. Br J Clin Pharmacol 1989;27: 329-335

15 Jonas WB, Dillner DK. Protection of mice from tularemia infection with ultra-low, serial agitated dilutions prepared from Francisella tularensis-infected tissue. J Sci Explor 2000;14:35-52

16 Bracho G, Varela E, Fernández R, et al. Large-scale application of highly-diluted bacteria for Leptospirosis epidemic control. Homeopathy 2010;99:156-166

17 Bracho G, Varela E, Fernández R, et al. Large-scale application of highly-diluted bacteria for Leptospirosis epidemic control. Homeopathy 2010;99:156-166 